\title{
COVID-19 Racial Discrimination and Depressive Symptoms among Asians Americans: Does Communication about the Incident Matter?
}

\author{
Bongki Woo ${ }^{1}$. Jungmi Jun ${ }^{2}$
}

Accepted: 15 February 2021 / Published online: 6 March 2021

○ The Author(s), under exclusive licence to Springer Science+Business Media, LLC part of Springer Nature 2021

\begin{abstract}
Since the outbreak of COVID-19, Asians in the US have experienced a spike of racism. The goal of this paper is to examine the association between racial discrimination amid COVID-19 pandemic and depressive symptoms among Asian subgroups and to test whether communications about the incident with various sources moderate this relationship. Data come from an online survey conducted among 245 Asian Americans. Multiple linear regression analyses were conducted. COVID-19 racial discrimination was positively associated with depressive symptoms, and this association did not vary between Chinese Americans and other Asian subgroups. Communications with a spouse/partner buffered the mental burden of racial discrimination. Those who shared their experience in online ethnic communities displayed stronger depressive symptoms than who did not. These results suggest the potential benefit of communication with a spouse/partner in mitigating the mental burden of discrimination and call for more online mental health support for Asians.
\end{abstract}

Keywords COVID-19 · Racial discrimination · Asian Americans · Depressive symptoms · Communication about discrimination

\section{Background}

Since the outbreak of COVID-19, there has been a spike of anti-Asian American racial discrimination and violent attacks. As of May 2020, Asian Pacific Policy and Planning Council (A3PCON) received over 1800 reports of racial discrimination associated with COVID-19 across the United States [1]. The reports of depression and anxiety symptoms among Asian Americans have increased sevenfold during the COVID-19 outbreak compared to 2019 [2]. The current climate of growing hostility against Asians and racial tensions calls for investigations on factors that can mitigate the

Bongki Woo

woob@mailbox.sc.edu

Jungmi Jun

junj@mailbox.sc.edu

1 College of Social Work, University of South Carolina, 1512 Pendleton Street, Room 307, Columbia, SC 29208, USA

2 School of Journalism and Mass Communications, College of Information and Communications, University of South Carolina, 800 Sumter Street, Room 306, Columbia, SC 29208, USA deleterious effects of racial discrimination amid COVID-19. The present study investigated how COVID-19 related racial discrimination is associated with depressive symptoms and the role of communication about the incident in moderating the negative mental health effects of discrimination.

Stress research paradigm and extant literature shows that racial discrimination is a chronic social stressor that negatively impacts racial minorities' mental health status, such as depressive symptoms [3-6]. Previous research suggests that covert discrimination (e.g., being treated with less courtesy or respect than others) is more prevalent than overt discrimination (e.g., being afraid of, called name, or threatened) among older Asian adults [7]. However, since the COVID-19 outbreak, more Asian Americans may face overt discrimination as reported to the $\mathrm{A} 3 \mathrm{PCON}$; the vast majority of reported incidents were overt discrimination, including verbal harassment and physical assaults [1]. Hence, it is likely that Asian who experienced racial discrimination during the pandemic may suffer from severe depressive symptoms, even after taking their previous experiences of racial discrimination into account. The present study specifically focuses on the mental effect of perceived everyday discrimination (i.e., frequent and recent events that focuses 
primarily on assaults to one's character) occurred before and after the COVID-19 pandemic, given that previous research suggest that everyday discrimination has more pronounced mental effects on the targeted individuals compared to major lifetime discrimination (e.g., major life events that impacts one's socioeconomic status) $[8,9]$.

Some ethnic groups within Asian Americans may experience particularly strong mental burden due to discrimination than others. According to the A3PCON report [1], over 40 percent of the COVID-19 racial discrimination incidents reports were from Chinese respondents, followed by other East Asians, including Koreans, Vietnamese, and Japanese. As the first outbreak of COVID-19 was reported in Wuhan, China and the racist and stigmatizing terms, such as "Chinese virus" or "Kung flu" or "Wuhan virus" have been consistently used to refer the virus [10], it is possible that Chinese Americans, in particular, feel stronger depressive symptoms from COVID-19 racial discrimination as it is directed to not only themselves but also their ethnic group memberships. Conversely, there may be no ethnic differences in the mental effect of discrimination since all Asian Americans are at risk of experiencing racial discrimination based on their appearance of 'looking like Chinese', regardless of their ethnicity.

\section{Theoretical Framework: The Role of Communications about Discrimination}

The influence of COVID-19 racial discrimination on mental health may vary according to the targeted individuals' responses to the incident. According to stress and coping theory, the way individuals deal with stress can either reduce or amplify the effects of stressful events, such as discrimination [11]. One of the common acts after experiencing discrimination is to discuss the incident with others $[12,13]$. A growing body of research suggests that social support gained from communication with others can help racial minority members become more resilient to future acts of discrimination [13-15]. In particular, targeted individuals of racism can fulfill their needs of informational/emotional support and self/group esteem by talking to others [14]. Disclosure of discrimination experiences can also help the targeted individuals to receive adequate supports from others that promote a sense of security and can mitigate the detrimental effects of discrimination $[12,13,16]$.

The potential buffering effects of conversations about discrimination may vary by its sources, whom the targeted individual talk with. For example, previous studies found that general perceived support from family mitigate the worse psychological distress and depression associated with unfair treatment $[17,18]$. Whereas, support from friends have mixed findings in that some previous research found buffering effect of peer support to mitigate the negative mental effects of discrimination $[19,20]$, while a few studies did not find its significant moderating effect $[17,18]$. Moreover, support provided by specific family members may play a different role, given that the relationships between spouses and parents, for example, vary [21]. Accordingly, spousal support was found to buffer the distress above and beyond supports from other family members in the context of unfair treatment [21], calling for more investigations on communications with various sources of support.

In our study, we investigated the buffering effects of communication about COVID-19 racial discrimination incidents with four different sources: spouse or partner, friends, online ethnic communities, and mental health professionals. As suggested in the previous findings [21, 22], the intimate and committed nature of the romantic relationships may buffer the mental burden of COVID-19 discrimination. Though the literature does not show consistent results on the buffering effects of supports from friends [17-20], it is possible that the peer network may provide a sense of connectedness and support for the targeted individuals during the COVID-19 pandemic when in-person interaction has been limited with the stay-home orders. In addition, by sharing discrimination experiences in online ethnic communities, targeted individuals can recognize that discrimination is a shared experience with their co-ethnics and find useful guidance or resources. Previous research found that social network with same-ethnic friends [23] or those who shared social group identity, such as sexual orientation [20], buffer the negative influence of discrimination on mental health. It is possible that online co-ethnic communities may play a similar role, especially since the pandemic has accelerated online communications. Lastly, discussing discrimination with mental health professionals or counselors can help individuals receive professional support and clinical services that can help their stress coping processes.

We conducted an online survey and collected data from Asian Americans who reported having experienced racial discrimination since the COVID-19 outbreak. The objectives of our study were to investigate the extent to which (1) perceived COVID-19 racial discrimination is associated with depressive symptoms above and beyond previous racial discrimination, (2) the relationship between COVID-19 racial discrimination and depressive symptoms vary by Asian ethnicity, (3) the role of communication about COVD-19 discrimination with various sources in buffering depressive symptoms.

\section{Methods}

\section{Data Collection}

We recruited participants via an online survey panel (Qualtrics) to collect data across the country in May 2020. Before 
entering to the survey questionnaire on Qualtrics platform, we asked participants to identify their race. Of the 1548 panels invited by Qualtrics, 858 respondents selected Asian as one of the multiple race options given (i.e., White, Asian, African American, Native Hawaiian or Pacific Islander, American Indian or Alaska Native). Then, we asked if they had experienced racist/xenophobia attacks, discrimination, harassment, and/or microaggression since the outbreak of COVID-19 because of their Asian race or ethnicity. Nearly $33 \%$ or 283 people reported to have experienced COVID-19 discrimination and voluntarily participated in the survey. After excluding incomplete or poor responses, a total of 245 responses were analyzed. Note that the race and ethnicity were self-identified, and the survey questionnaire was provided in the English language only. This study received the approval of the institutional review board, where the authors are affiliated.

\section{Measures}

\section{Depressive Symptoms}

Participants responded to the 20-item Center for Epidemiologic Studies of Depression (CES-D) Scale to assess the depressive symptom preceding one week [24]. Examples of the items included how often the respondent "thought [their] life had been a failure" and how often the respondent "felt that everything [they] did was an effort." The possible responses for each item ranged from 0 (rarely or none of the time) to 3 (most or all of the times). The scale demonstrated high internal consistency with an alpha coefficient of 0.92 . We used the sum of 20 items as an indicator of depressive symptoms.

\section{COVID-19 and Previous Racial Discrimination}

To measure COVID-19 racial discrimination, the respondents were asked to rate their experiences and perceived severity of different incidents of "being discriminated related with COVID-19." Following this lead-in question, we asked 10 types of racial discrimination and asked respondents to rate their experiences on each item. Examples of these items included "someone physically attacked me because of my race/ethnicity," "I was told to go back to my country because of my race/ethnicity," and "I was rejected from a service because of my race/ethnicity." The possible responses for each item ranged from 0 (did not happen) to 4 (it happened; very high severity). The scale displayed high internal consistency with an alpha coefficient of 0.93. The COVID-19 racial discrimination scale was calculated based on the mean of all responses.

In addition, the participants responded to the same ten questions that asked about the type and severity of discrimination that they had experienced before the COVID19 outbreak. The scale displayed high internal consistency with an alpha coefficient of 0.95 . We calculated the mean score of all responses.

\section{Communication about Discrimination and the Sources}

Participants responded to questions that asked how frequently they have talked or shared about their COVID-19 related discrimination experience with (1) spouse or partner, (2) friends, (3) online community for their ethnic group, and (4) mental health professionals or counselors. The possible responses for each item ranged from 1 (never) to 5 (very often).

\section{Asian Ethnicity}

The respondents self-identified themselves as Chinese, Korean, Japanese, Vietnamese, Filipino, or other Asian ethnic groups.

\section{Covariates}

We included a number of socioeconomic and demographic covariates that could potentially confound the association between racial discrimination and depressive symptoms: age in years, gender (female, male), education attainment (some college or less, college degree or more), household income ( $\$ 39,999$ or less; $\$ 40,000$ to $\$ 99,999, \$ 100,000$ or more), and nativity (whether born in the U.S. or not).

\section{Data Analysis}

The first set of analyses describes the means (percentages) and dispersion of the variables used in this study. Next, to examine the first research objective, multivariate linear regression analyses were conducted to assess the association between COVID-19 racial discrimination and depressive symptoms adjusting for previous racial discrimination and covariates (Model 1). Then, to investigate the second research objective, multivariate linear regression analyses were conducted to test the moderating effects of Asian ethnicity on the relationship between COVID-19 racial discrimination and depressive symptoms (Model 2). To test the third research objectives, we tested the association between different sources of communication and depressive symptoms (Model 3) and the moderating effects of communication about the incident on the relationship between COVID-19 racial discrimination and depressive symptoms (Model 4). For all models, we checked OLS regression assumptions, finding that all of them are met, including multi-collinearity (all VIF values were below 2.5 ), the normality of residuals, and linearity. Additionally, 
the correlation coefficient between COVID-19 and previous discrimination measures was 0.61 , indicating a moderate positive correlation. As over $98 \%$ of the study participants provided complete data, we used listwise deletion to deal with missing data. All analyses were conducted using Stata 15.1.

\section{Results}

Table 1 shows sociodemographic characteristics and descriptive statistics for depressive symptoms, COVID-19 related and previous racial discrimination, communication about COVID-19 racial discrimination with four sources, and ethnicity for the entire study sample. A mean of depressive symptoms was 22.69 , indicating that the study participants, on average, had either at least 11 of the 20 symptoms in the CES-D scale with a moderate persistence for the preceding one week or a majority of the symptoms for shorter periods of time. A mean of 2.40 was reported for our measure of COVID-19 racial discrimination, indicating that respondents experienced moderate to severe levels of discrimination.

Table 2 reports the results of multivariate linear regression analyses for the associations between racial discrimination, ethnicity, and depressive symptoms. According to Model 1, both COVID-19 racial discrimination $(b=4.40$, $p<0.001)$ and previous racial discrimination $(b=3.05$, $p<0.001$ ) were positively associated with depressive symptoms. There was no significant association between Asian ethnicity and depressive symptoms. Model 2 results show that the interaction terms between COVID-19 and ethnicity did not reach statistical significance, indicating the effects of discrimination do not vary between Chinese Americans and other Asian subgroups.

Table 3 displays the analyses results of the associations between COVID-19 racial discrimination, communication about discrimination with others, and depressive symptoms. Model 3 results show that all discussions with four different sources regarding COVID-19 racial discrimination were not statistically associated with depressive symptoms. Model 4 results suggests that communication about discrimination with a spouse or partner $(b=-1.17, p<0.05)$ and in online ethnic communities $(b=1.67, p<0.05)$ significantly moderate the relationship between COVID-19 racial discrimination and depressive symptoms. These results indicate that frequent communication with a spouse or partner buffer the relationship between COVID-19 racial discrimination and depressive symptoms. Whereas, those who experienced severer COVID-19 racial discrimination and often disclosed it in online ethnic communities report greater depressive symptoms than those who did not. Other interaction terms examined were not statistically significant.
Table 1 Depressive symptoms, COVID-19 racial discrimination, communication about discrimination with others, and demographic characteristics of Asians in the United States, 2020

\begin{tabular}{ll}
\hline Variable & $\begin{array}{l}\text { Mean (Standard } \\
\text { Deviation) or Per- } \\
\text { centage }\end{array}$ \\
\hline Depressive symptoms & $22.69(11.32)$ \\
COVID-19 racial discrimination & $2.40(1.00)$ \\
Previous racial discrimination & $2.36(0.99)$ \\
Communication about COVID-19 racial & \\
discrimination & \\
With spouse/partner & $2.34(1.47)$ \\
With friends & $2.43(1.40)$ \\
In online ethnic communities & $1.77(1.16)$ \\
With mental health professionals & $1.69(1.15)$ \\
Ethnicity & \\
Chinese & $27.08 \%$ \\
Korean & $10.83 \%$ \\
Japanese & $8.75 \%$ \\
Vietnamese & $10.83 \%$ \\
Filipino & $13.75 \%$ \\
Other & $28.75 \%$ \\
Age (years) & $31.13(11.46)$ \\
Gender & \\
Female & $60.83 \%$ \\
Male & $37.17 \%$ \\
Nativity & \\
US born & $63.75 \%$ \\
Foreign born & $36.25 \%$ \\
Household income & \\
\$40,000 - 99,999 & $25.83 \%$ \\
Education & $47.08 \%$ \\
Come college or less & $27.08 \%$ \\
\hline & \\
\hline & \\
College or more & \\
\hline
\end{tabular}

The sample size was $n=240$

\section{Discussion}

Asian Americans have experienced spurred racial discrimination and violent attacks since the COVID-19 pandemic and have displayed strikingly stronger mental health concerns than before. In addition to the stress associated with the pandemic itself, Asian Americans carry additional mental burden of being targets of misinformation surrounding the virus, and many are exposed to improper terms for the virus stigmatizing their ethnicity or origin. The present study sample consists of Asian Americans who have experienced COVID-19 racial discrimination. Given the detrimental effects of racial discrimination on mental health $[4,5]$, our study sample displayed greater 
Table 2 Associations between racial discrimination before and after COVID-19, Ethnicity, and depressive symptoms among Asians in the United States, 2020

\begin{tabular}{lll}
\hline & \multicolumn{2}{l}{ Coefficient (95\% Confidence Interval) } \\
\cline { 2 - 3 } & Model 1 & Model 2 \\
\hline COVID-19 racial discrimination & $4.40^{* * *}[2.90,5.90]$ & $3.37 * *[1.13,5.61]$ \\
Previous racial discrimination & $3.05^{* * *}[1.50,4.60]$ & $3.01^{* * *}[1.43,4.60]$ \\
Ethnicity (ref: Chinese) & & \\
Korean & $2.70[-1.53,6.93]$ & $3.11[-9.00,15.21]$ \\
Japanese & $1.94[-2.53,6.41]$ & $-4.05[-14.69,6.58]$ \\
Vietnamese & $2.25[-1.93,6.44]$ & $-6.08[-17.76,5.60]$ \\
Filipino & $-0.72,[-4.61,3.95]$ & $-4.00[-14.86,6.86]$ \\
Other & $0.75[-2.45,3.95]$ & $-1.90[-9.70,5.90]$ \\
Two-way interaction: COVID-19 racial dis- & & \\
crimination & & $-0.23[-5.24,4.79]$ \\
$\times$ Korean & & $2.49[-1.45,6.42]$ \\
$\times$ Japanese & & $3.64[-1.16,8.44]$ \\
$\times$ Vietnamese & & $1.39[-2.79,5.57]$ \\
$\times$ Filipino & & $1.13[-1.85,4.10]$ \\
$\times$ Other & & \\
\hline
\end{tabular}

All models control for age, gender, nativity, household income, and education. These estimates are not shown for the sake of space but are available upon request

$* p<0.05 ; * * p<0.01 ; * * * p<0.001$

\begin{tabular}{lll}
\hline & \multicolumn{2}{l}{ Coefficient (95\% Confidence Interval) } \\
\cline { 2 - 3 } & Model 3 & Model 4 \\
\hline COVID-19 racial discrimination & $4.25^{* * *}[2.71,5.80]$ & $5.19^{* * *}[2.45,7.92]$ \\
Communication about COVID-19 racial discrimination & & \\
With spouse/partner & $-0.36[-1.46,0.74]$ & $2.35[-0.58,5.27]$ \\
With friends & $0.27[-0.92,1.46]$ & $1.57[-1.76,4.90]$ \\
In online ethnic communities & $-0.89[-2.36,0.58]$ & $-5.59 * *[-9.7,-1.48]$ \\
With mental health professional/counselor & $1.37[-0.17,2.90]$ & $1.72[-2.46,5.91]$ \\
Two-way interaction:COVID-19 racial discrimination & & \\
$\times$ talked with spouse/partner & & $-1.17 *[-2.3,-0.04]$ \\
$\times$ talked with friends & & $-4.68[-1.72,0.78]$ \\
$\times$ talked in online ethnic communities & & $1.67 *[0.27,3.06]$ \\
$\times$ talked with mental health professional & & $-0.02[-1.38,1.34]$ \\
\hline
\end{tabular}

All models control for previous racial discrimination, age, gender, nativity, country of origin, household income, and education. These estimates are not shown for the sake of space but are available upon request $* p<0.05 ; * * p<0.01 ; * * * p<0.001$
Table 3 Associations between COVID-19 racial discrimination, communication about discrimination with others, and depressive symptoms among Asians in the United States, 2020 depressive symptoms than reported in previous Asian American studies [25-27].

Consistent with the extant research [4, 5], our findings showed that Asian Americans who reported severer exposure to racial discrimination during the COVID-19 pandemic were more likely to experience stronger depressive symptoms. Specifically, we found that, even when considered racial discrimination happened before the pandemic, COVID-19 racial discrimination was a significant social stressor. Our findings also showed that there were no ethnic differences in the detrimental mental effects of COVID-19 discrimination, indicating that COVID-19 racial discrimination negatively impacts all Asian Americans not only Chinese Americans. This result calls for more or continuous efforts to fight the stigma that associates COVID-19 with Asian American populations.

Though there were no significant direct protective effects of communication with others, the effect of COVID-19 discrimination on depressive symptoms was found to be buffered by talking with a spouse or partner, but not with friends. This result is consistent with the previous findings that proximal source of social support, such as family or spouse, 
mitigates mental health apprehensions against unfair treatment $[17,18,21]$. While a previous study found that spousal support buffered the psychological distress associated with general unfair treatment - but not with racial discrimination -, our findings indicate the spousal support can provide effective buffering effects in the context of COVID-19 racial discrimination. With the rapid increase of violent attacks and social distancing during the pandemic, it is possible that the bond shared in the intimate partnership has become more crucial when coping with racial discrimination.

Our finding suggests that those who shared their COVID19 racial discrimination experiences in online ethnic communities displayed stronger depressive symptoms. This result could be attributed to at least two factors. First, those who share their discrimination experiences with online ethnic groups may be more frequent internet and social media users. Racial and ethnic minority online users may be more exposed to the increasing blatant online racism targeting Asians during the COVID-19 pandemic [28] or may experience derogatory comments as they share their discrimination experiences online, which in turn can lead to severer mental burden. Second, although online ethnic communities may provide platforms to share the discrimination experiences, it may lack adequate information or support to help the targeted individuals. More quality resources for Asian Americans on the online ethnic communities and other social/ digital platforms are needed to help them cope with racial discrimination amid the COVID-19 pandemic. Although several Asian American advocate groups collect incident reports of COVID-19 discrimination and provide toolkits for confronting and correcting the racially charged terms for the virus [29], there is scarce information on strategies to cope with the stressful life events and enhance the physical and mental wellbeing for Asian American communities.

The lack of buffering effect of talking about COVID-19 racial discrimination with mental health professionals can be attributed to the sudden interruptions or changes in the treatment processes (e.g., a sudden shift of in-person sessions to online sessions) when physical distancing was required during the pandemic. In addition, culture-related barriers (e.g., stigma associated with mental illness, unfamiliarity with Western mental health services) among Asian Americans to initiate and continue mental health treatment may provide additional explanations on the null finding [30-32]. Western mental health services that involve open and intimate communications may conflict with cultural values of Asian American, which prevent sharing personal emotions or family matters with strangers to avoid having their name viewed poorly by others or losing face [30, 31]. Given some Asians' tendency of feeling face loss or shame of disclosing personal emotions (e.g., distress caused by racial discrimination) to therapist who is a stranger [33], it is possible that the targeted individuals feel reluctant to fully disclose themselves to providers or terminated treatments early especially in the virtual environment. Furthermore, the mental health impact of COVID-19 may take months or longer time to manage and requires concerted effort not just from mental health providers but from the health care system at large [34]. Our finding echoes the needs to incorporate Asian Americans as one of the vulnerable populations and to develop effective virtual mental health services (e.g., synchronous telemedicine services, self-help services) for the population $[35,36]$.

\section{Limitations}

The findings of this study should be considered in light of some limitations. First, the present study is based on crosssectional data, which precludes causal inferences about associations between variables. Second, Qualtrics-recruited sample were reported to be closest to a national probability sample on most demographic variables, including race and ethnicity, as compared to other online panels (e.g., Facebook, MTurk) [37]. However, online surveys using convenience samples recruited through commercial internet panels come with limitations, such as the respondent quality, attentiveness, and cooperativeness attributed to incentives.

Second, our study relied on retrospective self-report of discrimination before and after the COVID-19 outbreak. Longitudinal data that have collected data before the COVID-19 pandemic can yield new insights about the extent to which racial discrimination before and after the pandemic jeopardizes the mental health of the targeted individuals. Third, our measure of talking about racial discrimination to others was based on the single-item measure. Though the use of the single item to capture the disclosure to others is consistent with previous research of discrimination, future research will benefit by testing the various motivations behind the disclosure (e.g., to confirm that it was an act of discrimination, to seek help to cope with stress).

\section{New Contribution to the Literature}

Despite these limitations, the present study is one of the first to document the negative mental burden of the COVID-19 racial discrimination among Asians across the U.S. and the factors that can modify it. As most existing Asian American literature has focused on the quality or size of general support network in investigating buffering effects of social support, there has been limited understanding of the extent to which the actual conversations about discrimination experience with specific sources of potential support can mitigate the mental burden of racial discrimination. The present study identified significant moderating effects of communication with a spouse or partner and in online ethnic communities in the association between COVID-19 racial discrimination and depressive symptoms. 


\section{Conclusion}

The present study suggests that encouraging quality discussion of racial discrimination experiences with spouse or partner can mitigate the detrimental mental health effects of COVID-19 racial discrimination. Also, there are needs of further investigations on how COVID-19 racial discrimination has been discussed in online Asian ethnic communities. Beyond providing places to share the discrimination experiences, online ethnic communities can serve an important venue to co-create and distribute resources for coping of racism and menta health specific to Asian Americans. We also call for more culturally competent mental health services for Asian American communities that are easily accessible via social and digital platforms during the pandemic.

Acknowledgement This research was funded by the Office of the Vice President for Research at the University of South Carolina.

Funding This work was supported by University South Carolina Office of Research.

\section{References}

1. Jeung R. Incidents of Coronavirus discrimination, April 30 May13, 2020: a report for A3PCON and CAA. Stop AAPI Hate. Los Angeles, CA Asian Pacific Policy Plan. Counc. Chinese Affirm. Action. 2020. Available from: http://www.asianpacificpol icyandplanningcouncil.org/wp-content/uploads/STOP_AAPI_ HATE_Report_4_30_20-5_13_20.pdf. Accessed 15 June 2020.

2. National Center for Health Statistics. Percentages of selected mental health indicators for adults aged 18 and over, by race and Hispanic origin: United States, January-June 2019. 2020. Available from: https://www.cdc.gov/nchs/data/nhis/earlyrelease/ERmen talhealthbyrace-508.pdf. Accessed 15 June 2020.

3. Anderson KF. Diagnosing discrimination: stress from perceived racism and the mental and physical health effects. Sociol Inq. 2013;83:55-81.

4. Gee GC, Spencer M, Chen J, Yip T, Takeuchi DT. The association between self-reported racial discrimination and 12-month DSMIV mental disorders among Asian Americans nationwide. Soc Sci Med. 2007;64:1984-96.

5. Gee GC, Ro A, Shariff-Marco S, Chae D. Racial discrimination and health among Asian Americans: evidence, assessment, and directions for future research. Epidemiol Rev. 2009;31:130-51.

6. Chae DH, Lee S, Lincoln KD, Ihara ES. Discrimination, family relationships, and major depression among Asian Americans. J Immigr Minor Health. 2012;14:361-70.

7. Chan K. The association of acculturation with overt and covert perceived discrimination for older Asian Americans. Soc Work Res. 2020;44:59-71.

8. Ayalon L, Gum AM. The relationships between major lifetime discrimination, everyday discrimination, and mental health in three racial and ethnic groups of older adults. Aging Ment Health. 2011;15:587-94.

9. Kessler RC, Mickelson KD, Williams DR. The prevalence, distribution, and mental health correlates of perceived discrimination in the United States. J Health Soc Behav. 1999;1:208-30.
10. Somvichian-Clausen A. Trump's use of the term "Chinese Virus" for coronavirus hurts Asian Americans, says expert. Hill. 2020; Available from: https://thehill.com/changing-america/respect/ diversity-inclusion/489464-trumps-use-of-the-term-chinese-virus -for. Accessed 15 June 2020.

11. Lazarus RS. Coping theory and research: past, present, and future. Psychosom Med. 1993;55:234-47.

12. McLaughlin KA, Hatzenbuehler ML, Keyes KM. Responses to discrimination and psychiatric disorders among Black, Hispanic, female, and lesbian, gay, and bisexual individuals. Am J Public Health. 2010;100:1477-84.

13. Brondolo E, Ver Halen NB, Pencille M, Beatty D, Contrada R. Coping with racism: a selective review of the literature and a theoretical and methodological critique. J Behav Med. 2009;32:64-88.

14. Hanasono LK, Chen L, Wilson SR. Identifying communities in need: examining the impact of acculturation on perceived discrimination, social support, and coping amongst racial minority members in the United States. J Int Intercult Commun. 2014;7:216-37.

15. Finch BK, Vega WA. Acculturation stress, social support, and self-rated health among Latinos in California. J Immigr Health. 2003;5:109-17.

16. Ruggiero KM, Taylor DM, Lydon JE. How disadvantaged group members cope with discrimination when they perceive that social support is available. J Appl Soc Psychol. 1997;27:1581-600.

17. Mossakowski KN, Zhang W. Does social support buffer the stress of discrimination and reduce psychological distress among Asian Americans? Soc Psychol Q. 2014;77:273-95.

18. Tummala-Narra $\mathrm{P}$, Alegria M, Chen CN. Perceived discrimination, acculturative stress, and depression among South Asians: mixed findings. Asian Am J Psychol. 2012;3(1):3.

19. Grossman JM, Liang B. Discrimination distress among Chinese American adolescents. J Youth Adolesc. 2008;37:1-11.

20. Yoshikawa H, Alan-David Wilson P, Chae DH, Cheng J-F. Do family and friendship networks protect against the influence of discrimination on mental health and HIV risk among Asian and Pacific Islander gay men? AIDS Educ Prev. 2004;16:84-100.

21. Rollock D, Lui PP. Do spouses matter? discrimination, social support, and psychological distress among Asian Americans. Cult Divers Ethn Minor Psychol. 2016;22(1):47.

22. McNeil S, Fincham FD, Beach SRH. Does spousal support moderate the association between perceived racial discrimination and depressive symptoms among African American couples? Fam Process. 2014;53:109-19.

23. Ikram UZ, Snijder MB, de Wit MAS, Schene AH, Stronks K, Kunst AE. Perceived ethnic discrimination and depressive symptoms: the buffering effects of ethnic identity, religion and ethnic social network. Soc Psychiatry Psychiatr Epidemiol. 2016;51:679-88.

24. Radloff LS. The CES-D scale: a self-report depression scale for research in the general population. Appl Psychol Meas. 1977;1:385-401.

25. Kim E. Multidimensional acculturation attitudes and depressive symptoms in Korean Americans. Issues Ment Health Nurs. 2009;30:98-103.

26. Li Z, Hicks MHR. The CES-D in Chinese American women: construct validity, diagnostic validity for major depression, and cultural response bias. Psychiatry Res. 2010;175:227-32.

27. David EJR. A colonial mentality model of depression for Filipino Americans. Cult Divers Ethn Minor Psychol. 2008;14:118.

28. Schild L, Ling C, Blackburn J, Stringhini G, Zhang Y, Zannettou S 2020 "Go eat a bat, chang!": an early look on the emergence of sinophobic behavior on web communities in the face of Covid-19. arXiv Prepr arXiv200404046.

29. Nguyen M. COVID-19 toolkit strategies to defeat Asian American racial profiling and xenophobia. Organization of Chinese Americans. 2020. https://static1.squarespace.com/static/5e84f1ce44 
c90b6e8e673212/t/5ed65bf3d19b544bdd15b998/1591106558 969/OCA+COVID-19+Chapter+Toolkit+2020-05-21+compl ete.pdf. Accessed 15 June 2020.

30. Leong FTL, Lau ASL. Barriers to providing effective mental health services to Asian Americans. Ment Health Serv Res. 2001;3:201-14.

31. Tung WC. Cultural barriers to mental health services among Asian Americans. Home Health Care Manag Pract. 2011;23:303-5.

32. George S, Duran N, Norris K. A systematic review of barriers and facilitators to minority research participation among African Americans, Latinos, Asian Americans, and Pacific Islanders. Am J Public Health. 2014;104:e16-31.

33. Zane N, Yeh M. The use of culturally-based variables in assessment: studies on loss of face. Asian Am Ment Health. 2002. https ://doi.org/10.1007/978-1-4615-0735-2_9.

34. Rajkumar RP. COVID-19 and mental health: a review of the existing literature. Asian J Psychiatr. 2020;52:102066.
35. Zhou X, Snoswell CL, Harding LE, Bambling M, Edirippulige S, Bai $\mathrm{X}$, et al. The role of telehealth in reducing the mental health burden from COVID-19. Telemed e-Health. 2020;26:377-9.

36. Liu S, Yang L, Zhang C, Xiang Y-T, Liu Z, Hu S, et al. Online mental health services in China during the COVID-19 outbreak. Lancet Psychiatry. 2020;7:e17-8.

37. Boas TC, Christenson DP, Glick DM. Recruiting large online samples in the United States and India: Facebook, mechanical turk, and qualtrics. Polit Sci Res Methods. 2020;8:232-50.

Publisher's Note Springer Nature remains neutral with regard to jurisdictional claims in published maps and institutional affiliations. 